\title{
Modeling of ITER TF cooling system through 2D thermal analyses and enthalpy balance
}

\author{
Francesca Cau ${ }^{\mathrm{a}}$, Ruggero Forte ${ }^{\mathrm{b}}$, Alfredo Portone ${ }^{\mathrm{a}}$ \\ ${ }^{a}$ Fusion for Energy, F4E, Barcelona, Spain \\ ${ }^{b}$ Dipartimento di Energia, Ingegneria dell'Informazione e Modelli Matematici (DEIM), Università di Palermo, Viale \\ delle Scienze, Palermo, Italy
}

\begin{abstract}
The winding pack of the ITER Toroidal Field (TF) coils is composed of 134 turns of Nb3Sn Cable in Conduit Conductor (CICCs) wound in 7 double pancakes and cooled by supercritical helium (He) at cryogenic temperature. The cooling of the Stainless Steel (SS) case supporting the winding pack is guaranteed by He circulation in 74 parallel channels.
\end{abstract}

A 2D approach to compute the temperature distribution in the ITER TF winding pack is here proposed. The TF is divided in 32 poloidal segments, for each segment the corresponding 2D model is built and a thermal analysis is performed applying the corresponding nuclear heating computed with MCNP code considering the latest design updates, such as thickness increase of the blanket shield module. The Heat Transfer coefficient (HTC) of the He flowing in the CICC and in the cooling channels of the SS case is computed with Dittus Boelter correlation at the nominal inlet pressure of $6 \mathrm{bar}$. The He is assumed to enter the coil at $4.5 \mathrm{~K}$ in the lower terminal junction, and then the bulk temperature in all the CICCs in each of the 32 segments is calculated by means of enthalpy balance between segments, considering the actual direction of He circulation, i.e. clockwise or counter-clockwise in neighboring pancakes. The He properties needed to compute the HTC, such as viscosity, specific heat and thermal conductivity, are also varied using the same strategy. With these assumptions, He temperatures close to $5.7 \mathrm{~K}$ are computed, due to the high values of nuclear heating (which is estimated as high as $21.58 \mathrm{~kW}$ in the $18 \mathrm{TF}$ ). In the paper, the methodology is presented and the results are discussed in detail. Further parametric analyses are also presented to show the impact of the inlet temperature and of the nuclear heating on the temperature distribution.

\section{Introduction}

Heavy heat loads act on the TF coil system during operation, such as 1) the nuclear heating due to the plasma neutrons flux [1] 2) eddy currents in the metallic structures during plasma transients and instabilities [2] 3) thermal radiation from the thermal shield 4) AC loss (hysteresis and coupling) in the superconductors [3].

As stated in the recent STAC report [1], the value of the nuclear heating on the TF system reaches $21.58 \mathrm{~kW}$, with an upper bound of around $26 \mathrm{~kW}$, if the uncertainties due to statistical error of Monte Carlo simulations, to fusion power measurements and the ones related to nuclear data are taken into account.

The eddy currents in one TF coil case and supports can reach $0.17 \mathrm{MW}$ during normal scenario and 6.5 MW during plasma instabilities [2]. While the nuclear heating is constant during plasma flat top, the Joule losses develop during plasma transients and have a limited duration. However, these losses are so large that can compromise the integrity of the system.

There are two cooling systems for the TF coils, one for the WP, the other for the TF case and structures. The cooling circuit of the TF WP is made by the circulation of helium in the CICC. Each Double Pancake (DP) has the helium feed and return connection at the lower terminal junction, in which helium is driven to flow into two opposite flow directions, clockwise and anticlockwise. The cooling circuit of the TF case (see Figure 1) is equipped with parallel cooling circuits that run in the poloidal direction grouped in parallel sets by inlet and outlet sub-manifolds [4]. The aim of the cooling of the case is to intercept the heat before it is conducted to the WP [1].

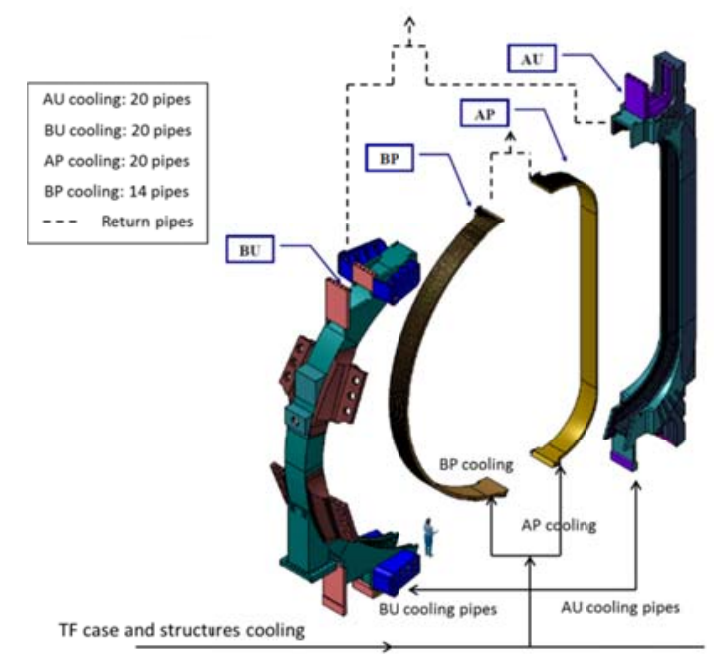

Figure 1 Cooling Layout of the TF case

We propose in this paper a simplified method which allows analyzing in very short time the response of the system to the neutronic loads. The analysis is based on a sequence of 2D steady state Finite Element (FE) thermal analyses of 32 poloidal segments of a single TF coil in which the own nuclear heating is applied. Starting from initial values of He bulk temperature and constant HTCs in all the circuits, an iterative procedure based on a series 
of runs is performed storing at the end of each run the powers extracted from each CICC and each TF Coil Cooling (TFCC) pipe. These values of extracted power are used to compute the new values of $\mathrm{He}$ bulk temperature by means of enthalpy balance (1) between the poloidal segments, fixing the He inlet temperature in the circuit to $4.3 \mathrm{~K}$ (and pressure 6 bar):

$$
H\left(T_{\text {out }}\right)=\frac{Q}{\dot{m}}+H\left(T_{\text {in }}\right)
$$

$\mathrm{Q}^{*}$ is the power extracted by helium in the previous segment, $\mathrm{m}$ is the helium mass flow rate, $\mathrm{H}\left(\mathrm{T}_{\text {out }}\right)$ is the Helium enthalpy at the outlet of each segment and $\mathrm{H}\left(\mathrm{T}_{\mathrm{in}}\right)$ is the helium enthalpy at the inlet of each segment. The actual direction of $\mathrm{He}$ circulation, i.e. clockwise or counter-clockwise in neighboring pancakes, is taken into account. With this approach, the heat conduction along the coil length is neglected. The main advantage of this calculation is that a complete temperature map in the WP of each segment is obtained, allowing further detailed thermal and mechanical analyses with the same ANSYS code. A similar methodology was already presented in [5] to compute the temperature distribution in the winding pack of the JT60 TF coil. In that analysis, transient simulations were performed, assuming a constant helium pressure. The helium temperature evolution was obtained computing in each segment the temperature of the jacket with a transient Finite Element Analysis and transferring this temperature to the adjacent segments for the subsequent run. In the present analysis, a steady state regime is considered and the pressure drop of the fluid is also taken into account. Of course the aim is not yet to compute precisely the He temperature in the cooling circuit and make evaluation concerning temperature margin, because only a steady state condition is modeled and the heat load due to eddy currents in the metallic structure and AC loss in the CICC is neglected, but it represents a first step in this direction.

\section{Model}

\subsection{Finite Element Model}

The model has been built with the commercial FE software ANSYS [6]. According to the poloidal segmentation reported in [1], 32 segments of the TF Coil are modelled dividing them in two sets: inboard segments (segments 28 to 32 and 1 to 12) and outboard segments (segments from 13 to 27). For simplicity, the transition regions between inboard and outboard legs are not modelled. For the TF WP cooling circuit, the He inlet and outlet are imposed at the segment 28 (see Figure 2). The inlet for the TF case cooling pipes is imposed at the same segment 28 , while the outlet at segment 12 . The 2D finite element models represents in very detailed way all the components of the WP, such as the Stainless Steel case, the cooling pipes of the case, the radial plates and the jackets of the CICCs (in SS [7]), the turn, ground and pancake insulation (Kapton Cyanate [8]). The case cooling pipes are in contact with the case trough a layer of resin, which is assumed in perfect contact with the steel. Contact elements have been placed between the WP and TF Coil Case Front, in order to simulate the gap between the WP and SS Case in the front part caused by magnetic forces in normal operation, and a thermal conductivity equal to zero has been imposed between them.

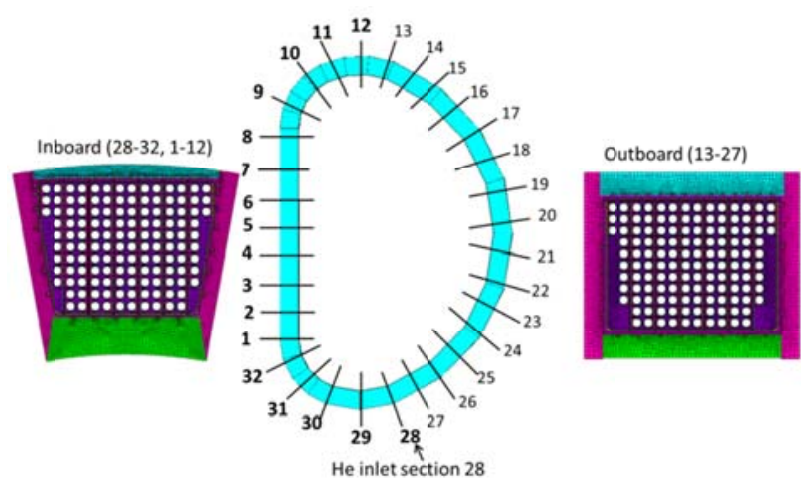

Figure 2 TF poloidal segments: inboard segments (in bold) and outboard segments.

\subsection{Loads}

As reported in [1], the "best estimate" of the nuclear heat load on the TF Coils is defined as $18.5 \mathrm{~kW}$ for the 18 TF Coils, while the more realistic "conservative" estimate is assessed to be equal to $21.57 \mathrm{~kW}$. The "conservative estimated" is deduced from the "best estimate" by applying appropriate correction factors in view of some modifications of both the model and the design. In particular, a reduction of the heat in the "inboard straight leg" is mostlly due to foreseen increase of the thickness of the blanket shield modules in the straight leg by $2 \mathrm{~cm}$. The reduction of the heat in the "outboard-bottom" region comes from an update of the model of the lower port, considered empty in the "best estimate". A first analysis is carried out applying the "best estimate" of the nuclear heating, while a second one the "conservative estimate" plus uncertainties (around 21\%) for a total heat load of around $26.10 \mathrm{~kW}$. The results of both analyses are then compared. The nuclear heat data for the 32 segments are reported in Table 1. The data in Table 1 have been normalized to each cross segment and applied as volumetric heat load to the $2 \mathrm{D}$ models. The values refer to 18 coils and the heating in the conductors is included. However, since the CICC are not modeled, the corresponding heat load is applied to the radial plates. In the following Figure 3, an example of volumetric nuclear heat load $\left[\mathrm{W} / \mathrm{m}^{3}\right]$ applied to segment \#5 (inboard) is shown. As can be noted, the load decreases while going from the plasma facing surface to the back part of the SS case.

Table 1 Best and conservative estimate of the nuclear heating

\begin{tabular}{ccc}
\hline TF Coil Regions & $\begin{array}{c}\text { Best } \\
\text { estimate }\end{array}$ & $\begin{array}{c}\text { Conservative } \\
\text { estimate }\end{array}$ \\
\hline Inboard straight leg (1-7) & $12.01 \mathrm{~kW}$ & $11.01 \mathrm{~kW}$ \\
Inboard top (8-11) & $1.58 \mathrm{~kW}$ & $3.38 \mathrm{~kW}$ \\
Outboard top (12-16) & $1.05 \mathrm{~kW}$ & $2.19 \mathrm{~kW}$ \\
Outboard equatorial (17-22) & $0.63 \mathrm{~kW}$ & $2.04 \mathrm{~kW}$ \\
Outboard bottom (23-28) & $2.65 \mathrm{~kW}$ & $2.33 \mathrm{~kW}$ \\
Inboard bottom (29-32) & $0.56 \mathrm{~kW}$ & $0.62 \mathrm{~kW}$ \\
TOTAL & $18.48 \mathrm{~kW}$ & $21.57 \mathrm{~kW}$ \\
\hline
\end{tabular}

\subsection{Boundary Conditions}


The external surfaces of the case have been considered as adiabatic surfaces. Convection has been imposed on all the internal surfaces of the CICC jackets and TFCC pipes. The Heat Transfer Coefficient has been computed with the Dittus-Boelter correlation (2) [9] taking into account the hydraulic parameters in Table 2.

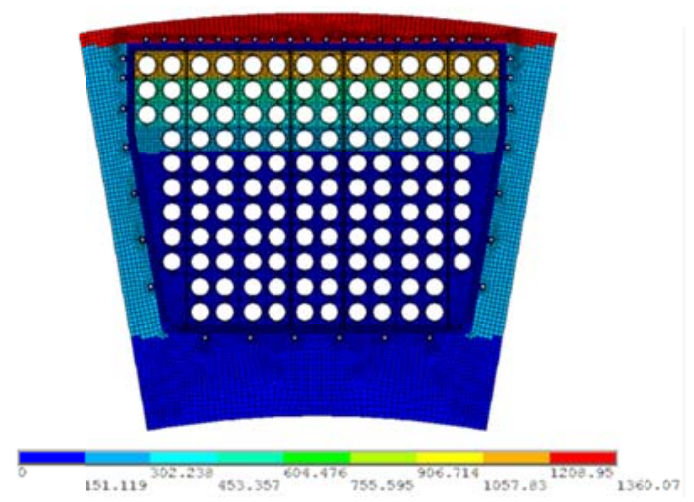

Figure 3 Volumetric nuclear heating $\left[\mathrm{W} / \mathrm{m}^{3}\right]$ applied to FE model of the inboard (segment \#05)

$$
\begin{aligned}
& \operatorname{Re}=\frac{4 \dot{m}}{\pi D_{h} \mu} \\
& \operatorname{Pr}=\frac{\mu c_{p}}{k} \\
& h=\left(0.023 \cdot \operatorname{Re}^{0.8} \cdot \operatorname{Pr}^{0.4}\right) \cdot\left(k / D_{h}\right)
\end{aligned}
$$

where Re is the Reynolds number, Pr is the Prandtl number, $\mathrm{k}$ is the thermal conductivity and $\mathrm{D}_{\mathrm{h}}$ is the hydraulic diameter. The $\mathrm{He}$ enthalpy and the $\mathrm{He}$ properties used for the HTC computation are calculated as function of temperature and pressure basing on the data in [10].

Table 2 Hydraulic parameters

\begin{tabular}{ccc}
\hline & Hydraulic Diameter & Mass flow rate \\
\hline CICC & $0.34 \mathrm{~mm}$ & $0.0079 \mathrm{~kg} / \mathrm{s}$ \\
\hline TFCC Pipe & $7.8 \mathrm{~mm}$ & $0.0027 \mathrm{~kg} / \mathrm{s}$ \\
\hline
\end{tabular}

\subsection{Pressure Drop}

The pressure drop evaluation has been implemented with two different approaches in the two helium cooling circuits. The correlation (3) from [11] has been used for the WP cooling circuit, assuming that the pressure drop in steady state is the same in the bundle region and in the central channel [13].

$$
\mathrm{f}_{\mathrm{B}}=\left(\frac{1}{\varphi}\right)^{0.742} \cdot\left(0.0231+\frac{19.5}{\mathrm{Re}_{B}}\right)^{0.7953}
$$

where $\mathrm{Re}_{\mathrm{B}}$ is the bundle Reynolds number and $\varphi(=0.34)$ is the void fraction. $\operatorname{Re}_{\mathrm{B}}$ is computed using the bundle helium mass flow rate $(\sim 35 \%$ of the total flowing in CICC [11] [12]), a hydraulic diameter of $\approx 0.34 \mathrm{~mm}$ (four times the fluid area of the bundle divided by the wetted perimeter) and the helium properties depending both upon temperature and pressure.
For the TF case cooling circuit the Petukhov correlation (4), used with good approximation for smooth pipes in turbulent regime, [13] has been used.

$$
f_{p}=\left(0.79 \log \left(R e_{\text {pipes }}\right)-1 . .64\right)^{-2}
$$

The maximum CICC and case pipes channel length is $380 \mathrm{~m}$ and $\approx 17 \mathrm{~m}$, respectively.

\subsection{Results of the Analyses}

The procedure used can be summarized by the following steps:

1. A first run with initial values of $\mathrm{He}$ bulk temperature equal to $4.3 \mathrm{~K}$ (at pressure of 6 bar) in the whole circuits is performed, applying to the 32 segments the corresponding nuclear heat load. In each run, the power extracted from each CICC and each pipe is computed.

2. The enthalpy at the outlet of the segment, and hence the corresponding $\mathrm{He}$ temperature, is computed; new heat transfer coefficients in each single CICC and TF case cooling pipe are also determined.

3. The thermal analysis of the 32 segment is repeated, using the updated HTC computed in the previous iteration. The powers extracted from each CICC and each pipe are computed again and the loop restarts from point (2).

The computational time of each iteration is approximately $445 \mathrm{~s}$. The analysis finishes when the bulk temperatures converge to a final value, i.e. when the maximum percentage difference between the bulk temperatures of two subsequent iterations is lower than $0.1 \%$. The maximum number of iterations to reach convergence is around 26 . It has to be pointed out that the analysis is done considering a steady state, therefore all the transient phenomena are neglected. This is a strong assumption because the ITER functioning is pulsed, and the method as it is cannot be used to make assessment on Tcs. However, this procedure can be used to perform some comparative studies and optimization of the structure from both thermal and mechanical point of view. In fact, the temperature map computed in the WP with this approach is more accurate than assuming a uniform bulk temperature of $4.3 \mathrm{~K}$ in all the CICC.

The final values of HTCs are in the range between 240 and $325 \mathrm{~W} / \mathrm{m}^{2} \mathrm{~K}$ for the CICC and between 580 and $900 \mathrm{~W} / \mathrm{m}^{2} \mathrm{~K}$ for the pipes of the TF case circuit.

Around $70 \%$ of the total heat is applied to the SS case and it is evacuated by the cooling pipes of the case.

The bulk temperature along the length of pancake $14^{\text {th }}$ is shown in Figure 4. In this pancake, the temperature in the high field region close to the plasma is the highest, in fact the coolant arrives at the straight leg already warmer because it has already passed along the outboard region. The continuous line in Figure 4 is obtained applying the conservative estimate of the nuclear heating $(26.1 \mathrm{~kW})$ neglecting pressure drop, the triangles correspond to the temperature profile obtained with the same heat load taking also into account the 
pressure drop, while the thickest continuous line is obtained with the best estimate of the nuclear heating $(18.5 \mathrm{~kW})$. As can be noted, the curves of the temperature considering a total power of $26 \mathrm{~kW}$ with and without considering pressure drop perfectly overlap.

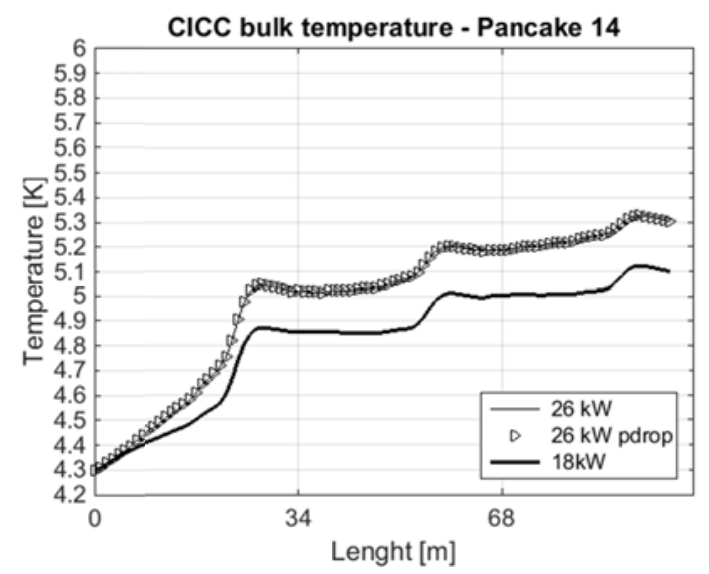

Figure 4 Temperature along the $14^{\text {th }}$ pancake

In the conservative assumption of steady state regime the outlet temperature of the Helium reaches $5.3 \mathrm{~K}$ and $5.1 \mathrm{~K}$ applying the conservative and the best estimate of the nuclear heating, respectively. These temperatures are above the target limit of $5.0 \mathrm{~K}$ and in agreement with the results obtained with the thermo-hydraulic code VINCENTA [1]. However, due to the assumptions made, the location of the maximum He temperature is not in the innermost turns as computed in VINCENTA, but in a region close to the outlet. The temperature jumps in Figure 4 are due to the enthalpy increase in correspondence of the inboard leg, which is the most loaded region of the TF. Starting from the $6^{\text {th }}$ layer of conductors from the plasma facing surface (around 200 $\mathrm{m})$, the heat load is so low that the He starts to be cooled by the radial plates in the outboard region, and its temperature remains stable.

The maximum pressure drop obtained in the WP cooling circuit is around 0.6 bar, which is lower than the specifications [4] probably due to the assumption on the friction factor and on the mass flow rate in the bundle region. In the $\mathrm{TF}$ case cooling circuit the effect of pressure drop on the bulk temperature is negligible $(\approx 0.01$ bar $)$. This minimum variation of pressure does not affect the temperature profile. In fact, the dotted and the continuous lines overlap.

As far as the jacket is concerned, the peak temperature is located in correspondence the last turn of the $2^{\text {nd }}$ and $13^{\text {th }}$ pancake, depending on the segment, and it is around $5.5 \mathrm{~K}$. The temperature map obtained in the most loaded segments (segment \#05) is shown in Figure 5. The peak temperature of the SS case is located in the uppermost corners, which are far from the cooling pipes. As far as the cooling pipes of the TF SS case are concerned, the outlet temperature is slightly above $6 \mathrm{~K}$. In Figure 6, the bulk temperature of the inboard pipes located in the front part of the case is shown for segments 1 to 6 (seg. 1 to seg. 6), which are the most loaded segments (see Figure 2 and Table 1). As can be noted, the highest temperature is found in the corner pipes, in agreement with Figure 5.
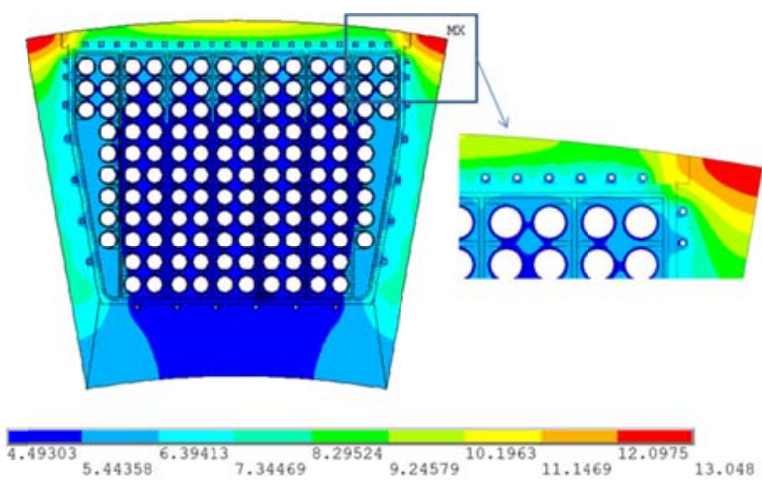

Figure 5 Temperature map obtained in the most loaded segments (segment \#05) -26.1 kW, no pressure drop

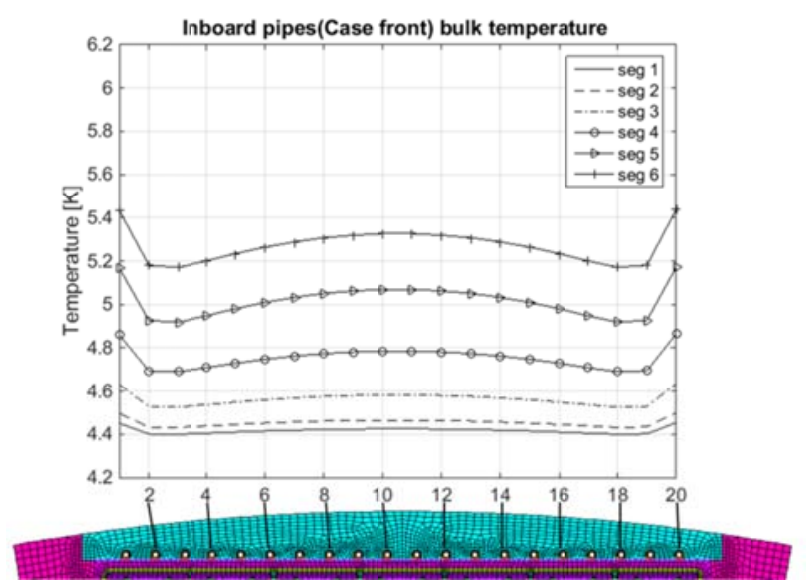

Figure 6 Bulk temperature of the inboard leg pipes in the front part of the case

\section{Conclusions}

A simplified methodology to compute the temperature distribution in the ITER TF winding pack is proposed. The approach consists in coupled 2D thermal analyses and 1D hydraulic model of the Helium flow. The procedure has been entirely developed in ANSYSAPDL. The advantage of this procedure is that it is fast, it allows to compute the temperature map in the WP of each segment taking into acicount the He temperature evolution. However, it must be highlighted that the steady state condition is a strong assumption and it might have an important impact on the results of this analysis. Therefore, a transient approach is suggested in order to have more accurate results. A further development of the work consists in improve the model to make it transient adding the heat load due to plasma instabilities.

\section{Disclaimer}

This publication reflects the views only of the author, and Fusion for Energy and ITER cannot be held responsible for any use which may be made of the information contained therein.

\section{References}

[1]. IC/STAC-18/2.3 "Report on progress on evaluation of neutronic issues", April 2015 
[2]. F. Cau, D. Bessette, G. D'Amico, A.Portone, P. Testoni et a. "Joule Losses in the ITER Cold Structures During Plasma Transients", IEEE Transactions on Applied Superconductivity 26 (4) (2016)

[3]. L. Bottura, P. Bruzzone, J. B. Lister, C. Marinucci and A. Portone, "Computation of the AC loss in the ITER magnets during fast field transient", IEEE Transaction on Applied Superconductivity 17 (2) (2007)

[4]. ITER Design Description Document (DDD) 11-2, "TF Coils and Structures", September 2009

[5]. C. Portafaix, P.Barabaschi, F. Gauthier, et al., "Development of the Pseudo 3D Thermo Hydraulic Tool TACOS: Application to JT-60SA TF Coils Design Optimization", IEEE Transactions on Applied Superconductivity 20 (3) (2010)

[6]. ANSYS user manual, release 17.0

[7]. Annex to Design Requirements and Guidelines Level 1 (DRG1), "Superconducting Material Database", Article 5. Thermal, Electrical and Mechanical Properties of Materials at Cryogenic Temperatures, N 11 FDR 42 0107-05 R 0.

[8]. "Thermal contact - Final report", Cecile Foucher de Brandois, private communication, April 2015

[9]. M. Lewandoska, L. Malinowski, "Transverse Heat Transfer Coefficient in the Dual Channel ITER TF CICCs. Part III: Direct Method of Assessment", Cryogenics 73 (2016)

[10]. Cryosoft version 2.0, February 1997

[11]. R. Zanino, P.Bruzzone, L. Savoldi Richard, "A critical assessment of the pressure drop design criteria for the conductors of the ITER Magnets" AIP Conference Proceeding 823 (1765) (2006)

[12]. S. Nicollet et al., "Calculations of pressure drop and mass flow distribution in the toroidal field model coil of the ITER project", Cryogenics 40 (2000)

[13]. Frank P. Incropera, David P. Dewitt "Fundamentals of Heat and Mass Transfer", Wiley, 1985 